$\underline{\text { Jordi Tudela Fernández }^{1}}$

\title{
El Sistema para la Autonomía y la Atención a la Dependencia en Cataluña
}

SUMARIO: 1. GÉNESIS DEL PROGRAMA PRODEP.- 2. MISIÓN DEL PROGRAMA PRODEP.- 3. ESTRUCTURA ORGANIZATIVA Y MEDIOS. 3.1. CONSEJO Director. 3.2. Consejo Asesor. 3.3. Consejo de Participación. 4. IMPleMENTACIÓN NORMATIVA DEL SISTEMA. 5. NOVEDADES DE LA LEY 12/2007, DE 11 DE OCTUBRE, DE SERVICIOS SOCIALES. 6. INTEGRACIÓN DE SERVICIOS. 7. PRINCIPIOS DE EL MODELO- LA ATENCIÓN A LAS DEPENDENCIAS EN CATALUÑA. 8. IMPLEMENTACIÓN DEL SISTEMA. 8.1. Sistema de INFORMACión A LOS PROFESIONALES SOBRE EL PROCESO. 8.2. Sistema de información a la CiUdAdanía SObre la DependenCia. 8.3. AportaCIÓN A LAS ENTIDADES LOCALES DE LOS MEDIOS NECESARIOS PARA IMPLEMENTAR EL SisTeMa. 8.4. Creación de UN SiSTEMA OPERATIVO E INFORMÁtico. 8.5. Creación De la herramienta para el Conocimiento de la dependencia. 8.6. DesplieGUE DEL PROCESO DE RECONOCIMIENTO DEL GRADO Y NIVEL DE DEPENDENCIA. 9. EXPERIENCIA NOVEDOSA-DESARROLLO DE LA ATENCIÓN A LA DEPENDENCIA EN CATALUÑA 2008-2011. 10. EL PRESUPUESTO POR PROGRAMAS DE LA GENERALITAT 2008 RESPECTO DEL PROGRAMA DE ATENCIÓN A LA GENTE MAYOR CON DEPENDENCIA. 11. NORMATIVA CATALANA DE DESPLIEGUE DE LA LEY 39/2006, DE 14 DE DICIEMBRE. 12. CONCLUSIONES.

${ }^{1}$ Director del ProdeP, el Programa para el Impulso y la ordenación de la promoción de la autonomía personal y la atención de las personas con dependencia de la Generalitat de Cataluña. 


\section{GÉNESIS DEL PROGRAMA PRODEP}

El 31 de mayo de 2005, el Gobierno de la Generalitat acordó la creación del Programa Prodep, de carácter interdepartamental, con la finalidad de impulsar y ordenar la promoción de la autonomía personal y la atención a las personas con dependencias.

Este programa fue presentado públicamente el 22 de junio y entró en funcionamiento el mes de septiembre de 2005.

El programa Prodep está adscrito al Departament d'Acció Social i Ciutadania de la Generalitat de Cataluña.

\section{MISIÓN DEL PROGRAMA PRODEP}

Diseñar un modelo de organización y atención a las personas en situación de dependencia que sea funcional, a partir de la base de una acción integrada entre servicios sociales y de salud y de base territorial.

\section{ESTRUCTURA ORGANIZATIVA Y MEDIOS}

Este programa está compuesto por tres órganos colegiados rectores: Consejo Director, Consejo Asesor y Consejo de Participación.
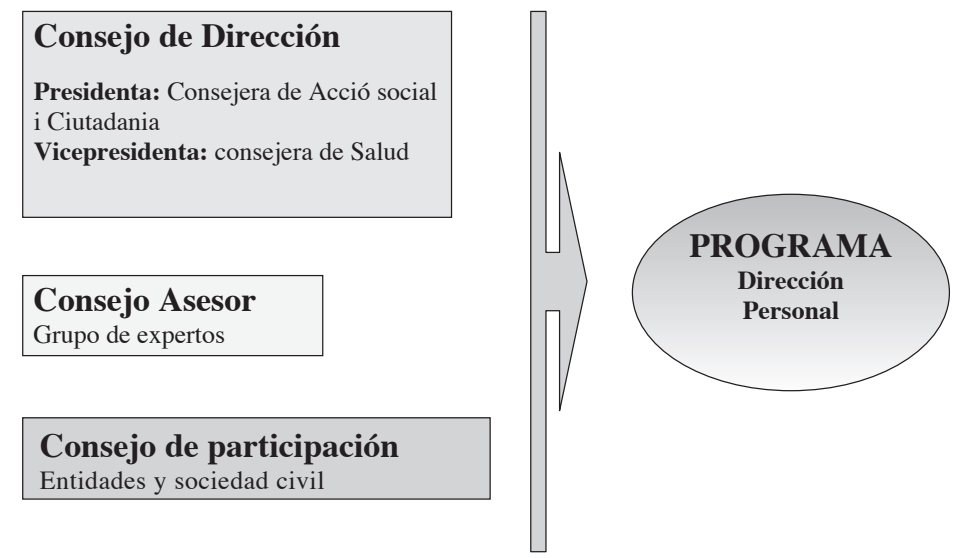

3.1. El Consejo Director es un órgano colegiado que tiene como funciones diseñar, informar y proponer al Govern las políticas de servicios sociales y de salud en relación a la protección de las personas con dependencias y en el marco de las competencias de la Generalitat de Cataluña. Es un órgano compuesto de 14 miembros y presidido por la Consellera d'Acció So- 
cial i Ciutadania, con la vicepresidencia de la Consellera de Salud (como programa interdepartamental que es). Además cuenta entre sus vocales con representantes de Departament d'Economia i Finances, de la Federación de Municipios de Cataluña, de la Asociación Catalana de Municipios y Comarcas, de la Diputación de Barcelona, de la Cruz Roja, así como de representantes de los Departamentos de Presidencia y Vicepresidencia.

3.2. El Consejo Asesor es un órgano colegiado integrado por personas expertas de diferentes ámbitos, entre los que destacan: salud (economía de salud, servicios de salud comunitarios, salud mental), servicios sociales y sociosanitarios, servicios para la gente mayor, gestión de organizaciones y evaluación de la calidad.

Este órgano tiene como funciones el asesoramiento técnico y la propuesta de medidas para la elaboración del modelo del sistema de atención a las dependencias (además de aquellas que le encarguen el Consejo Director o la Dirección del programa).

3.3. El Consejo de Participación es un órgano colegiado de implicación activa de la sociedad civil en el desarrollo de la Ley de Promoción de la Autonomía y Atención a las Personas con Dependencia (LEPA). Es un espacio de reflexión, colaboración y participación de los diversos sectores sociales implicados en la atención a las personas en situación de dependencia y para la promoción de la autonomía personal. A día de hoy esta ha sido la actividad del Consejo:

- Aprobación del Reglamento de Funcionamiento Interno.

- Celebración de reuniones sectoriales con las diferentes entidades.

- Elaboración de un plan de trabajo a partir de las sugerencias y propuestas de las entidades.

- Realización de jornadas informativas sobre el despliegue de la LEPA con las diferentes entidades.

- Elaboración y puesta en marcha de las bases reguladoras para la ampliación del Plenario, para la constitución de la Comisión Permanente y para la organización de los diversos grupos de trabajo del Consejo de Participación.

El Consejo de Participación está integrado por representantes de:

- Organizaciones de personas usuarias y familiares.

- Organizaciones prestadoras de servicios.

- Corporaciones profesionales.

- Organizaciones sindicales.

- Entidades del tercer sector social.

- Centros educativos. 
- Federación de mutualidades.

- Federación de cajas de ahorros.

Miembros del Consejo de Participación:

- Coordinadora d'Usuaris de la Sanitat (CUS).

- Consell General de la Gent Gran.

- Federació Catalana d'Associacions de Familiars de Malalts Mentals (FECAFFAM).

- Federació Catalana Pro Persones amb Retard Mental (APPS).

- COCEMFE Catalunya.

- Foro Vida Independent.

- Federació d'Associacions de Familiars de Malalts Mentals d'Alzheimer i d'altres Demències de Catalunya.

- Unió Catalana d'Hospitals (UCH).

- Consorci d'Hospitals de Catalunya (CHC).

- ACES.

- Associació Catalana de Recursos Assistencials (ACRA).

- FEATE.

- FERS.

- Fòrum de Salut Mental.

- Comitè Català de Representants de Persones amb Discapacitat (COCARMI).

- Creu Roja Catalunya.

- Coordinadora de Centres de Profunds de Catalunya (CCPC).

- Col.legi de Diplomats/des en Treball Social.

- Col.legi Oficial de Psicòlegs de Catalunya.

- Col.legi de Fisioterapeutes.

- Associació Professional de Terapeutes Ocupacionals de Catalunya (APTOC).

- Associació de Treballadors Familiars

- UGT.

- CC.OO.

- Taula d'entitats del tercer sector de Catalunya.

- Federació Catalana de Caixes d'Estalvi.

- Federació de Mutualitats.

\section{IMPLEMENTACIÓN NORMATIVA DEL SISTEMA}

El 1 de enero de 2007 entra en vigor la Ley 39/2006, de 14 de diciembre, de promoción de la autonomía personal y atención a las personas en 
situación de dependencia. Estas son las principales características que incorpora la Ley:

- Reconocimiento del derecho universal y subjetivo de la ciudadanía a la promoción de la autonomía personal y atención de las personas en situación de dependencia.

- Creación del Sistema para la Autonomía y Atención a la Dependencia (SAAD) de carácter público, con la participación de las diferentes administraciones, en el ejercicio de sus competencias.

- Estructuración de la dependencia en tres grados y cada grado en dos niveles. Para atender a las situaciones de necesidad derivadas del grado y nivel, la Ley prevé prestaciones económicas así como servicios. La Ley establece preferencia por los servicios y, en el caso que no sea posible el servicio, se instaura la prestación económica.

- Definición del copago: los beneficiarios de las prestaciones de dependencia participarán en la financiación de las mismas, según el tipo y coste del servicio y su capacidad económica personal.

Posteriormente el Parlament de Catalunya aprueba la Ley 12/2007, de 11 de octubre, de servicios sociales que desarrolla la aplicación en Cataluña el Sistema de Autonomía y Atención a la Dependencia (SAAD) mediante la implementación del Sistema Catalán de Autonomía y Atención a la Dependencia (SCAAD). A partir de este momento ya se hablará de un único sistema que incorpora las dos leyes. Es decir, se ha elaborado un modelo único de organización para atención de la personas en situación de dependencia.

\section{NOVEDADES DE LA LEY 12/2007, DE 11 DE OCTUBRE, DE SERVICIOS SOCIALES}

- Dota el sistema de cobertura universal.

- Reconoce específicamente el derecho subjetivo de acceso a los servicios sociales.

- Presenta una descripción detallada de los derechos y los deberes de las personas en relación con los servicios sociales.

- Introduce la Cartera de Servicios Sociales como un instrumento para asegurar el acceso a las prestaciones garantizadas.

- Regula el papel de los profesionales en los servicios sociales.

- Se adapta a les necesidades actuales de envejecimiento de la población, de crecimiento demográfico acelerado, de nuevas "bolsas" de pobreza, de cambios en el mercado laboral, etc. 


\section{INTEGRACIÓN DE SERVICIOS}

Puesto que es necesario desarrollar una acción integrada y compactada entre los servicios sociales y los servicios de salud, el programa integra:

1) La participación de los Departamentos d'Acció Social i Ciutadania y Salut.

2) Las administraciones locales con competencias en servicios sociales de atención primaria.

3) El gobierno de los servicios sanitarios territoriales.

\section{PRINCIPIOS DEL MODELO LA ATENCIÓN A LAS DEPENDENCIAS EN CATALUNYA}

No se crea una tercera red o tercer sistema. Se trata de una acción compactada-integrada entre los Servicios Sociales y los de Salud. Con ello se pasa de un proceso administrativo a un modelo profesionalizado responsable; acercando así el acceso al ciudadano y resultando, por tanto, un modelo de base territorial. Se garantiza la continuidad asistencial, el acompañamiento en el proceso y se prioriza la atención en la comunidad, cosa que supone el mando conjunto entre las administraciones públicas competentes en colaboración con la sociedad civil.

\section{IMPLEMENTACIÓN DEL SISTEMA}

La puesta en marcha durante el 2007 del sistema de atención a las personas con dependencia tuvo un coste inicial de 13 millones de euros. Para ello se llevaron a cabo las siguientes acciones:

- Sistema de información a los profesionales sobre el proceso.

- Organización de 98 conferencias, coloquios dirigidos a ayuntamientos y consejos comarcales (regidores/as y jefes/as de Área de Servicios Sociales).

- Las referentes del Prodep, en el territorio, han llevado a cabo funciones de asesoramiento técnico para el diseño y validación de los procesos, además de dar información básica a profesionales y entidades.

- Jornadas con profesionales del sector. 
- Sistema de información a la ciudadanía sobre la dependencia.

- Puesta en marcha de un sistema de información a la ciudadanía mediante los teléfonos: 012 y 900300500 para hacer accesible la información sobre el despliegue de la LEPA.

- Puntos de información en los servicios sociales de atención primaria y en las áreas básicas de salud.

- Creación de la página web www.gencat.catldependencia donde pueden encontrarse: los objetivos de la Ley, tabla explicativa de los grados de dependencia, formulario de autoevaluación sobre la situación de dependencia, boletín de la dependencia, calendario de aplicación de las prestaciones, puntos de información, datos de contacto del Prodep, preguntas más frecuentes, enlaces y novedades.

- Publicación y difusión en el territorio de dípticos y material informativo sobre la dependencia.

- Jornadas:

- Coloquios en entidades de personas con discapacidad, de gente mayor y otros colectivos.

- Participación, mediante ponencias, en mesas redondas y coloquios, en los cuales se han difundido los contenidos y las ventajas de la Ley de la dependencia entre entidades del Tercer Sector.

- Participación en medios de comunicación escritos, radio y TV de ámbito catalán.

- Aportación a las entidades locales de los medios económicos y humanos necesarios para implementar el sistema:

- Contratación de 23 referentes del Prodep en el territorio

- 61 convenios con ayuntamientos y 39 convenios con consejos comarcales para ampliar el número de trabajadores y trabajadoras sociales: 105 en todo el territorio durante el primer año.

- Contratación de 21 empresas públicas para que gestionen los 23 servicios de valoración de la dependencia (SEVAD) distribuidos por toda Cataluña, los cuales realizan los informes sobre la situación de dependencia utilizando el instrumento acordado por la LEPA.

- Creación de un sistema operativo informático que permite gestionar la base de datos de la dependencia y los procesos de recogida de datos, 
la emisión de resoluciones y el envío de las resoluciones a las entidades locales para la realización de los PIA's.

- Creación de la herramienta del conocimiento para la dependencia. El objetivo es centralizar toda la información relacionada con la dependencia, prevista en diversos aspectos de la Ley. El sistema está pensado para que cualquier persona con conocimientos básicos de usuario pueda clasificar i buscar los contenidos.

- Instrucción 2/2007, de 30 de julio de 2007, emitida por la Secretaría General del Departament d'Acció Social i Ciutadania mediante la cual se establecen los criterios generales para tramitar el procedimiento de reconocimiento de la situación de dependencia y el procedimiento de elaboración del Programa Individual de Atención (PIA). El objeto de esta Instrucción es el de establecer los criterios generales para tramitar, en el marco del SAAD, los siguientes ámbitos:

1) El procedimiento de reconocimiento de la situación de dependencia.

2) El procedimiento de elaboración del Programa Individual de Atención (PIA): elaboración, propuesta de resolución, resolución, impugnación y plazos.

3) Valoración de la capacidad económica y determinación de la participación de la persona beneficiaria en el coste de los servicios.

4) Gestión de los datos personales

5) Régimen jurídico general

Órganos competentes: la competencia formal se delimitará de acuerdo a la normativa vigente y los instrumentos que correspondan de delegación y encargo de gestión en vigor, en particular:

- Decreto 115/2007, 22 de mayo, por el cual se determinan los órganos de la Generalitat competentes para aplicar la Ley 39/2006, de 14 de diciembre.

- Los que determinen los decretos del Gobierno de la Generalitat y las órdenes dictadas por la Conselleria d'Acció Social i Ciutadania (DASC), así como los acuerdos de delegación de competencias en favor de las entidades locales de Cataluña.

- Los convenios formalizados entre el DASC y entidades locales catalanas para la elaboración del Programa Individual de Atención (PIA).

- El DASC se reserva la potestad normativa para regular la totalidad del procedimiento y la representación y defensa jurídica ante los juzgados y tribunales, en las demandas interpuestas en el ámbito regulado en este procedimiento. 
Fases del procedimiento:

1) La solicitud tiene los requisitos generales previstos en la Ley 30/1992, de 26 de noviembre, así como los requisitos específicos del artículo 5 de la Ley 39/2006, de 14 de diciembre. El modelo de solicitud está también disponible en la página web del DASC http://www.gencat.cat/benestar

Lugares de presentación: servicios territoriales i centrales del DASC, oficinas de atención al ciudadano del Departament de Governació i Administracions Públiques, el Consorcio de Servicios Sociales de Barcelona, entidades locales (en caso de existir convenio), registros generales de otros departamentos de la Generalitat, Administración General del Estado y en el servicio de correos.

La fecha de recepción de la solicitud en el órgano competente determinará el inicio del cómputo del plazo para resolver y notificar. En el caso que falten documentos o datos se concederá un plazo de 10 días para subsanación de defectos. En el caso de incumplimiento de este plazo por parte del solicitante se le declarará desistido y se emitirá la correspondiente resolución.

Finalmente, el órgano competente remitirá el expediente al equipo de valoración que corresponda.

2) Valoración: el equipo de valoración citará a la persona solicitante para valorar su situación de necesidad por cualquier medio admisible en derecho y quedará constancia documentada en el expediente.

Se podrá suspender la tramitación de expediente cuando, por razones no imputables al interesado, no sea posible valorar la situación de dependencia en el entorno habitual del solicitante. La suspensión se acordará en supuestos como el de enfermedad o ingreso del interesado en una centro sanitario, ingreso temporal en un centro residencial o ausencias temporales del domicilio habitual por causas no imputables al solicitante.

La suspensión de la tramitación implicará la suspensión del plazo para resolver y notificar, durante un máximo de 3 meses, pasados los cuales se declarará la caducidad del procedimiento.

Para los casos de negativa, desacuerdo de fechas o problemas de localización y en procedimientos paralizados por causa imputable al interesado, excepto los supuestos descritos anteriormente, se podrá declarar la caducidad del procedimiento.

En el caso que el órgano valorador hubiera advertido al interesado por escrito y le hubiere concedido un plazo de tres meses para hacer los pasos para reiniciar el procedimiento, para el caso de incumplimiento de este plazo el órgano competente podrá resolver el archivo del expediente, con notificación a la persona interesada. 
- Evaluación, el equipo de valoración valorará la situación de dependencia de acuerdo con: el RD 504/2007, de 20 de abril; las directrices del Consejo Territorial; el convenio con la respectiva entidad colaboradora del DASC y la legislación de procedimiento administrativo.

- El dictamen será motivado e incluirá los antecedentes, los elementos esenciales de la valoración, la determinación del grado y nivel de dependencia que corresponda. Se ajustará a un modelo normalizado.

- El equipo de valoración remitirá el dictamen, acompañado del expediente completo, al órgano competente para resolver.

- La resolución: el órgano competente recogerá el dictamen elaborado incluido los antecedentes esenciales para la valoración. El plazo máximo para resolver y notificar la valoración de la situación de dependencia es de 3 meses, contando desde la entrada de la solicitud en el registro competente.

- Las resoluciones reguladas en este apartado podrán ser objeto de reclamación previa, de acuerdo con lo establecido en el artículo 71 de la Ley de Procedimiento Laboral. El órgano competente, el mismo que dictó la resolución inicial, resolverá y notificará la resolución sobre reclamación previa.

Estructura de los equipos de valoración (SEVAD): se ha dotado al sistema de 23 equipos de valoración (SEVAD), agrupados en 7 sectores territoriales. Se dispone de un equipo básico de valoración, por cada 350.000 habitantes, de 5 valoradores. Cada equipo cuento con un/a valorador/a especializado/a en la valoración de niños y niñas entre 0 y 3 años. Estos equipos requieren una formación preferentemente en la licenciatura de medicina; en las diplomaturas de enfermería, terapia ocupacional, fisioterapia y otras titulaciones que como mínimo sean de grado medio.

Se ha formado a los equipos de valoración y a los/as profesionales que han de realizar el PIA. Se han organizado tres acciones formativas para personal valorador (185 personas), por la que respecta a la formación del personal que realiza el PIA se han efectuado 224 sesiones con 2.398 asistentes.

Funciones de los SEVAD:

- Preparación de la valoración, que consiste en la revisión de los informes de salud.

- Citación

- Valoración con un baremo de valoración de dependencia en domicilios o en centros.

- Revisión y asesoramiento de un equipo consultor. 
- Comisión de valoración que hace la propuesta de grado y nivel.

Comisión de valoración, el informe final: esta Comisión está formada por el equipo consultor, el/la coordinador/a y el/la referente. En la Comisión pueden participar representantes de la Administración y valoradores/as.

- Concluye sobre casos complejos: TMS, discapacidad intelectual, brotes, valoraciones de enfermedad sobrevenida, problemática social y/o emocional...

- Resuelve las propuestas de dictamen provisional

- Propone resoluciones de grado y nivel de situación de dependencia. Acta general con los acuerdos tomados y registro de cada caso.

\section{3) Elaboración del Programa Individual de Atención (PIA)}

- El órgano que ha dictado la resolución de valoración remitirá el expediente al órgano encargado de elaborar el PIA.

- Citación de la persona beneficiaria, el órgano competente citará por cualquier medio admisible en derecho a la persona beneficiaria. La fecha a partir de la cual la persona beneficiaria podrá ser citada estará indicada en la resolución de reconocimiento de la situación de dependencia.

- Elaboración del PIA, la propuesta será elaborada por las Áreas Básicas de Servicios Sociales en los términos establecidos en el convenio y/o normativa de coordinación aprobada por el Gobierno de la Generalitat.

- Se han formalizado convenios con ayuntamientos y entidades locales para la realización de Programas Individuales de Atención, así como para la ampliación y refuerzo de la atención primaria social.

- El Prodep se ha dotado de 23 referentes que son el puente de unión entre el Prodep y el territorio.

- El PIA concretará las modalidades de intervención más adecuadas, de entre las reconocidas en la resolución de valoración, con la participación de la persona beneficiaria. Las modalidades de intervención se establecerán de acuerdo a los recursos disponibles.

- Contenido de la propuesta, los servicios y/o prestaciones económicas concretas que correspondan, de entre las reconocidas en la resolución de valoración.

- Valoración de la capacidad económica y determinación de la participación de la persona beneficiaria en el coste de los servicios. 
4) Resolución: los órganos competentes resolverán y notificarán el PIA, el plazo máximo para resolver y notificar la resolución del Programa Individual de Atención es de 3 meses, contando desde la fecha de incoación.

- Reclamación previa, las resoluciones reguladas en este apartado podrán ser objeto de reclamación previa, de acuerdo con lo establecido en el artículo 71 de la Ley de Procedimiento Laboral.

- Ejecución de la resolución del PIA, el acceso de la persona beneficiaria a las prestaciones económicas o a una plaza en el servicio y/o servicios establecidos por la resolución del PIA requerirá que el órgano administrativo correspondiente (Generalitat o entidad local, en el marco del convenio correspondiente) ordene el acto de ejecución material correspondiente.

- Durante este año se ha apoyado especialmente a la administración local en lo que respecta al aumento de la financiación de los entes locales para que realicen PIA.

— Inversión en las referentes del Prodep en el territorio de 990.000 euros.

- Inversión en el plan de apoyo a los entes locales de 3 millones de euros.

— Inversión en financiación de 166 trabajadores/as sociales de 5 millones de euros.

- Registrándose un apoyo total a la primaria en 2008 de casi 9 millones de euros.

Desde diciembre de 2007 hasta septiembre de 2008, el Departament d'Acció Social i Ciutadania ha realizado una inversión de 144 millones en la implantación del sistema catalán de promoción de la autonomía personal y atención a las personas con dependencia

\section{EXPERIENCIA NOVEDOSA}

Desarrollo de la atención a la dependencia en Cataluña 2008-2011 (Acuerdo estratégico para la internacionalización, la calidad de la ocupación y la competitividad de la economía catalana 2008-2011)

Este acuerdo entre el Gobierno de la Generalitat y el Consejo de Instituciones del Acuerdo Estratégico, que engloba a los principales agentes económicos i sociales (CC.OO. de Cataluña, UGT de Cataluña, Fomento del Trabajo, Pimec i Fepime) culmina un proceso de elaboración, diálogo y concertación con respecto al despliegue de la LEPA y la Ley catalana de servicios sociales. 
El Gobierno se compromete a impulsar las siguientes actuaciones:

- Reforzar los servicios sociales básicos, con la implantación del gestor de casos de alta dependencia, e incrementar la red de profesionales de los entes locales para la atención a la persona dependiente y a su familia, así como para la elaboración y el seguimiento de los planes individuales de atención (PIA).

- Incrementar los servicios de ayuda a domicilio hasta llegar a dar cobertura al 4\% de la población catalana mayor de 65 años i garantizar el derecho de atención mediante la red de atención a la dependencia y la vida autónoma. En la línea de universalizar el acceso a los servicios de teleasistencia, durante la legislatura se priorizarán aquellas personas que viven solas o en situación de desamparo.

- Definir los nuevos perfiles profesionales vinculados al ámbito de los servicios sociales y, de acuerdo con el Instituto Catalán de Calificaciones Profesionales, promover el sistema de acreditación de las competencias adquiridas mediante la formación y la experiencia laboral.

- En el marco de la Mesa Técnica de Gente Mayor, se podrán tratar con carácter consultivo los aspectos derivados del despliegue del sistema catalán de autonomía y atención a la dependencia que les corresponda.

- Concretar las necesidades de capital humano asociadas a los nuevos yacimientos de ocupación derivados de la Ley de servicios sociales y del sistema de atención a la dependencia en Cataluña. Esta tarea incluirá el desarrollo de planes de actuación, de la mano de las organizaciones sindicales y empresariales más representativas, para desarrollar la oferta necesaria que permita cubrir las necesidades de este sector.

\section{METAS}

\begin{tabular}{|c|c|c|c|c|}
\hline \multicolumn{1}{|c|}{ Indicadores del logro de la medida } & 2008 & 2009 & 2010 & 2011 \\
\hline $\begin{array}{c}\text { 1. Estimación del número de personas atendi- } \\
\text { das por la red de atención a la de-pendencia } \\
\text { y la vida autónoma }\end{array}$ & 50.035 & 77.049 & 98.792 & 103.840 \\
\hline $\begin{array}{l}\text { 2. Incremento del número de profesionales de } \\
\text { los servicios sociales de atención primaria }\left(^{*}\right)\end{array}$ & $\begin{array}{c}173 \mathrm{TS} \\
142 \mathrm{ES}\end{array}$ & $\begin{array}{c}173 \mathrm{TS} \\
142 \mathrm{ES}\end{array}$ & $\begin{array}{c}161 \mathrm{TS} \\
81 \mathrm{ES}\end{array}$ & $\begin{array}{c}161 \mathrm{TS} \\
81 \mathrm{ES}\end{array}$ \\
\hline $\begin{array}{l}\text { 3. Número de personas atendidas por los servi- } \\
\text { cios de ayuda a domicilio }\end{array}$ & 46.982 & 48.880 & 50.184 & 51.488 \\
\hline $\begin{array}{l}\text { 4. Media mensual de horas de atención domici- } \\
\text { liaria en Cataluña }\end{array}$ & 250.000 & 350.000 & 450.000 & 475.000 \\
\hline
\end{tabular}

$\left(^{*}\right)$ TS: trabajadores/as sociales

ES: educadores/as sociales 


\begin{tabular}{|c|c|c|c|c|c|}
\hline \multirow{2}{*}{$\begin{array}{c}\text { Presupuesto } \\
(\mathrm{M} €)\end{array}$} & \multicolumn{2}{|c|}{2008} & & 2 & 2 \\
\cline { 1 - 4 } & $\begin{array}{c}\text { Importe } \\
\text { incluido en } \\
\text { presupuesto } \\
\text { inicial }\end{array}$ & $\begin{array}{c}\text { Financiación } \\
\text { externa }\end{array}$ & 2009 & 2010 & 2011 \\
\hline Gasto & 671,91 & 128,36 & $1.080,36$ & $1.155,99$ & $1.213,79$ \\
\hline Inversión & 25,50 & 3,13 & 30,06 & 31,57 & 33,15 \\
\hline
\end{tabular}

Unidad responsable: Oficina de Planificación y Evaluación Económica, Programa para el impulso y la ordenación de la promoción de la autonomía personal y la atención a las personas en situación de dependencia (ProdeP), Instituto Catalán de Asistencia y Servicios Sociales (ICASS) y Gabinete Técnico de Acció Social i Ciutadania.

\section{EL PRESUPUESTO POR PROGRAMAS DE LA GENERALITAT 2008 RESPECTO AL PROGRAMA DE ATENCIÓN A LA GENTE MAYOR CON DEPENDENCIA}

Ley 16/2007, de 21 de diciembre, de presupuestos de la Generalitat de Cataluña para el 2008

\section{Departament d'Economia i Finances}

Unidad responsable: ICF Equipaments, SAU

\section{Diagnóstico de la situación:}

Necesidad de aumentar y mejorar los equipamientos en materia de servicios: una función básica del poder público es garantizar una adecuada prestación de los servicios, y por eso es necesario una infraestructura adecuada, en número suficiente y cumpliendo los requisitos mínimos legales exigidos.

Necesidad de centrar en una única empresa de titularidad pública la promoción y gestión de equipamientos de uso público.

\section{Misión:}

Promover y gestionar equipamientos para uso público de servicios a la comunidad. Construcción y gestión de inmuebles destinados a equipamientos públicos, poniéndolos a disposición de la administración correspondiente.

Objetivos estratégicos y operativos:

1. Promover y gestionar equipamientos para uso público.

1.1. Gestión y seguimiento de la construcción y posterior arrendamiento de los inmuebles destinados a equipamientos. 
2. Construcción y gestión de inmuebles destinados a equipamientos públicos, poniéndolos a disposición de la administración.

2.1. Gestión y seguimiento de la construcción y posterior arrendamiento de los inmuebles destinados a equipamientos.

Servicios/productos/actividades:

1. Construcción de centros

2. Arrendamiento de centros

3. Mantenimiento de los centros hasta su reversión en la administración correspondiente

Indicadores:

1. Centros en funcionamiento

2. Porcentaje presupuesto ejecutado de construcción de centros

\section{Departament de Política Territorial i Obres Públiques}

Unidad responsable: Gestió d'Infraestructures, SA (GISA)

Diagnóstico de la situación:

Con tal de optimizar los costes de funcionamiento y mejorar permanentemente la calidad de la ejecución por lo que respecta a costes, plazos, seguridad y medio ambiente, mediante acuerdo el gobierno ha encomendado a GISA obras del Departamento de Acción Social y Ciudadanía, incluidas en el presente programa.

El objeto social de Gisa es "proyectar, construir, conservar explotar y promocionar todo tipo de infraestructuras y edificaciones de terceros con las cuales la Generalitat haya acordado la construcción.

\section{Misión:}

La misión de la sociedad es contratar y ejecutar de forma eficiente y respetuosa con el medio ambiente las actuaciones encomendadas por los diferentes departamentos fomentando la concurrencia y la equidad y asegurando la transparencia, la calidad y la seguridad.

Objetivos estratégicos y operativos:

1. Ejecutar el programa de inversiones que nos ha sido encomendado

1.1. Optimizar los costes de funcionamiento

2. Mejorar permanentemente la calidad de los servicios prestados

2.1. Optimizar la calidad de la ejecución por lo que respecta a los costes, plazos, seguridad y medioambiente. 
Servicios/productos/actividades:

1. Mejora permanente en la calidad de la ejecución por lo que respecta a los costes, plazos, seguridad i medio ambiente

2. Gestión de obras y proyectos encomendados por el Departament d'Acció Social i Ciutadania

3. Obras de infraestructura de edificación

4. Estudios y proyectos asociados a las infraestructuras mencionadas.

\section{Indicadores:}

1. Número de obras a finalizar de atención a la gente mayor

2. Número de obras en ejecución de atención a la gente mayor

\section{Departament d'Acció Social i Ciutadania}

Unidad responsable: Gabinete y Secretaría General de Acción Social

\section{Diagnóstico de la situación:}

La mejora del bienestar de las personas mayores es uno de los objetivos prioritarios del Gobierno de la Generalitat de Cataluña, y la atención a la gente mayor con dependencia se configura como uno de los grandes retos de la política social europea de las próximas décadas, no únicamente en Cataluña, sino para el conjunto de la Unión Europea. El aumento del envejecimiento de la población catalana sitúa actualmente la tasa de personas mayores de 65 años en más del $16,5 \%$ de la población total.

El número de personas de más de 65 años es de 1.150.724, de las cuales 556.745, que suponen el $8 \%$ de la población general, son mayores de 75 años, y por tanto susceptibles de recibir servicios asistenciales especializados (datos de población 2005). Las proyecciones de población de Cataluña elaboradas por el IDESCAT prevén, a partir de una combinación selectiva de las hipótesis de evolución de los componentes de crecimiento demográfico, que el año 2010 la población mayor de 65 años representará casi el $19 \%$ de la población total, y llegará hasta un $24 \%$ de ésta el año 2030.

Este escenario hace necesario diseñar y llevar a cabo políticas de atención y promoción del bienestar de las personas mayores, y en concreto, de las personas mayores con dependencia, con el objetivo de facilitar las condiciones de vida que contribuyan a la conservación de la plenitud de sus facultades físicas y psíquicas, como también su integración social. 
La Ley estatal 39/2006, de 14 de diciembre, de promoción de la autonomía personal y atención a las personas en situación de dependencia (LAPAD), configura el cuarto pilar del estado del bienestar con la creación del Sistema de Autonomía y Atención a la Dependencia basado en el derecho universal, subjetivo y exigible. La implantación de este sistema es progresiva entre 2007 y 2015 y su gestión corresponde a la Generalitat de Cataluña en el marco de sus competencias exclusivas i cofinanciado con la Administración del Estado. Para el año 2007 las personas mayores destinatarias de este derecho subjetivo son las personas con un nivel de gran dependencia y se ha estimado su número en 21.600 personas.

El Departamento lo hace mediante el Instituto Catalán de Asistencia y Servicios Sociales (ICASS) por motivos de eficacia.

\section{Misión:}

Diseñar, regular y proveer los servicios y recursos asistenciales para la atención y promoción del bienestar de las personas mayores, y en concreto, de las personas mayores con dependencia, con el objetivo de facilitar las condiciones de vida que contribuyen a la conservación de la plenitud de sus facultades físicas y psíquicas, como también a su integración social.

\section{Objetivos estratégicos y operativos:}

1. Garantizar la atención a la gente mayor con dependencia con el fin de mejorar la calidad de vida de este colectivo.

1.1. Transferir al ICASS los recursos necesarios para complementar la financiación del programa de atención a la gente mayor con dependencia.

Servicios/productos/actividades:

1. Transferir al ICASS, con la periodicidad adecuada, la financiación correspondiente al programa de atención a la gente mayor con dependencia.

\section{Indicadores:}

1. Miles de euros transferidos al ICASS para políticas de atención a la gente mayor con dependencia. 


\section{Departament d'Acció Social i Ciutadania}

Unidad responsable: Instituto Catalán de Asistencia y Servicios Sociales

\section{Diagnóstico de la situación:}

La mejora del bienestar de las personas mayores es uno de los objetivos prioritarios del Gobierno de la Generalitat de Cataluña, y la atención a la gente mayor con dependencia se configura como uno de los grandes retos de la política social europea de las próximas décadas, no únicamente en Cataluña, sino para el conjunto de la Unión Europea. El aumento del envejecimiento de la población catalana sitúa actualmente la tasa de personas mayores de 65 años en más del 16,5\% de la población total.

El número de personas de más de 65 años es de 1.150.724, de las cuales 556.745, que suponen el 8\% de la población general, son mayores de 75 años, y por tanto susceptibles de recibir servicios asistenciales especializados (datos de población 2005). Las proyecciones de población de Cataluña elaboradas por el IDESCAT prevén, a partir de una combinación selectiva de las hipótesis de evolución de los componentes de crecimiento demográfico, que el año 2010 la población mayor de 65 años representará casi el 19\% de la población total, y llegará hasta un 24\% de ésta el año 2030.

Este escenario hace necesario diseñar y llevar a cabo políticas de atención y promoción del bienestar de las personas mayores, y en concreto, de las personas mayores con dependencia, con el objetivo de facilitar las condiciones de vida que contribuyan a la conservación de la plenitud de sus facultades físicas y psíquicas, como también su integración social.

La Ley estatal 39/2006, de 14 de diciembre, de promoción de la autonomía personal y atención a las personas en situación de dependencia (LAPAD), configura el cuarto pilar del estado del bienestar con la creación del Sistema de Autonomía y Atención a la Dependencia (SAAD) basado en el derecho universal, subjetivo y exigible. La implantación de este sistema es progresiva entre 2007 y 2015 y su gestión corresponde a la Generalitat de Cataluña en el marco de sus competencias exclusivas i cofinanciado con la Administración del Estado.

\section{Misión:}

Diseñar, regular y proveer los servicios y recursos asistenciales para la atención y promoción del bienestar de las personas mayores, y en concreto, de las personas mayores con dependencia, con el objetivo de facilitar las condiciones de vida que contribuyen a la conservación de la plenitud de sus facultades físicas y psíquicas, como también a su integración social. 
Objetivos estratégicos y operativos:

1. Favorecer que las personas mayores con dependencia permanezcan en el propio hogar y en su entorno habitual.

1.1. Otorgar ayudas económicas para el soporte a las personas con dependencia que reúnan los requisitos establecidos en el programa.

2. Mejorar la red asistencial destinada a las personas mayores con necesidades de atención especializada.

2.1. Lograr una cobertura de plazas residenciales con financiación pública de 2 por cada 100 personas de 65 años y más.

2.2. Incrementar el número de plazas de acogimiento diurno.

Servicios/productos/actividades:

1. Diseñar los programas de intervención para el logro de los objetivos planteados.

2. Hacer las gestiones para la promoción de la creación de plazas.

3. Hacer las actuaciones necesarias para la creación de plazas de titularidad pública y del Departamento.

4. Gestionar el incremento presupuestario para atender de forma eficiente la demanda no atendida.

5. Gestionar el incremento de precios para la compra de servicios.

6. Impulsar las actuaciones para el desarrollo del modelo rural.

7. Desarrollar el programa de atención a la dependencia.

8. Actualizar los criterios de análisis de necesidades y evaluar las respuestas de las mismas.

\section{Indicadores:}

1. Beneficiarios de una ayuda económica para personas dependientes en el marco del programa de soportes económico para gente mayor.

2. Plazas en residencias propias y concertadas (gente mayor)

3. Plazas residenciales colaboradoras (gente mayor)

4. Plazas residenciales con financiación pública por cada 100 personas mayores de 65 años.

5. Plazas de centros de día colaboradores para gente mayor.

6. Plazas de viviendas tuteladas propias y con centros colaboradores para gente mayor. 
7. Plazas concertadas de larga estada y hospital de día en el módulo social para gente mayor.

8. Usuarios atendidos por los PADES para personas dependientes.

9. Plazas en centros de día propios y concertados (gente mayor).

\section{NORMATIVA CATALANA DE DESPLIEGUE DE LA LEY 39/2006, DE 14 DE DICIEMBRE}

Ley 12/2007, de 11 de octubre, de servicios sociales (DOGC4990, de 18/10/07)

El artículo 166 del Estatuto de Autonomía de Cataluña atribuye a la Generalitat la competencia exclusiva en materia de servicios sociales.

El Sistema Catalán de Autonomía y Atención a la Dependencia (SCAAD).

En el marco del sistema público de servicios sociales, se configura una red de atención a la dependencia y la vida autónoma que comprende el conjunto de recursos, equipamientos, proyectos, actividades y prestaciones de servicios, económicas y tecnológicas en este ámbito de atención y protección, como Sistema Catalán de Autonomía y Atención a la Dependencia (SCAAD), a los efectos del despliegue y la aplicación en Cataluña del Sistema de Autonomía y Atención a la Dependencia (SAAD), creado con carácter general por la Ley del Estado 39/2006.

Así, las prestaciones en el ámbito de la atención a la dependencia y la vida autónoma se integran en el Sistema Catalán de Servicios Sociales, que llega a ser un sistema único e integral en Cataluña.

ORDEN ASC/55/2008, de 12 de febrero, por la que se establecen los criterios para determinar las compatibilidades y las incompatibilidades entre las prestaciones del Sistema Catalán de Autonomía y Atención a la Dependencia (SCAAD) y las prestaciones del Sistema Público de Servicios Sociales (SPSS) en el ámbito territorial de Cataluña. (DOGC 5075, de 21/02/08)

El Sistema Público de Servicios Sociales de Cataluña (SPSS) dispone, desde hace años, de un conjunto de programas para atender a las personas en situación de dependencia. El principio general de la Ley (artículo 31) es la incompatibilidad entre las prestaciones de análoga naturaleza y finalidad, sin perjuicio del nivel de protección vigente, por lo que procede regular los supuestos de incompatibilidad entre ambos sistemas.

$\mathrm{Al}$ respecto, las prestaciones de la Ley estatal suponen una mejora sobre la situación vigente, no tan sólo en la financiación, sino también en la protección jurídica, puesto que tienen carácter de derecho subjetivo. Por esta razón, el régimen de incompatibilidades considera prioritaria la conversión 
de las prestaciones vigentes de concurrencia, de carácter anual y limitadas por la disponibilidad presupuestaria, en las nuevas prestaciones de dependencia, garantizando, en su caso, el importe previo más favorable.

En todo caso, el régimen de incompatibilidades solamente afectará a las personas beneficiarias de las nuevas prestaciones de dependencia, de modo gradual, de acuerdo con el calendario de entrada en vigor de la Ley 39/2006, pero no afectará a las prestaciones del SPSS en vigor del resto de las personas dependientes, sin perjuicio de la limitación presupuestaria propia de las prestaciones de concurrencia.

ORDEN ASC/432/2007, de 22 de noviembre, por la que se regulan los precios públicos y el régimen de participación de laspersonas beneficiarias en la financiación de los servicios del Sistema para la Autonomía y la Atención a la Dependencia (SAAD) en el ámbito territorial de Cataluña. (DOGC 5017, de 27/11/07)

El artículo 33 de la Ley 39/2006, de 14 de diciembre, de promoción de la autonomía personal y atención a las personas en situación de dependencia, establece la participación de las personas beneficiarias de las prestaciones en la financiación de las mismas, de acuerdo con su capacidad económica.

En el caso de Cataluña, los precios públicos y las aportaciones de los beneficiarios de los servicios sociales están regulados por el Decreto 394/1996, de 12 de diciembre, por el que se establece el régimen de contraprestaciones de los usuarios en la prestación de servicios sociales y se aprueban los precios públicos para determinados servicios sociales prestados por la Generalitat de Cataluña. El Decreto fue actualizado por la Orden ASC/603/2006, de 21 de diciembre.

En concreto, la norma ordena los elementos necesarios para determinar el precio final del servicio, concretamente, a) el listado de los servicios, b) los precios públicos de referencia, c) los criterios de valoración de la capacidad económica de las personas beneficiarias y d) el régimen de participación de las personas beneficiarias en la financiación de los servicios, derivado de su capacidad económica.

Tal y como prevé el Decreto 394/1996, el régimen de participación de la persona beneficiaria en el coste del servicio garantiza un mínimo de ingresos, que variará en función de la tipología del servicio y se calculará teniendo en cuenta el índice de renta de suficiencia de Cataluña, tal y como prevé la disposición adicional 9. ${ }^{a}$ de la Ley 4/2007, de 4 de julio, de presupuestos de la Generalitat de Cataluña para 2007.

En consecuencia, la persona beneficiaria, descontados los ingresos garantizados, tan sólo abonará la renta disponible, en la que, por otro lado, 
también se aplicarán previamente las bonificaciones o reducciones correspondientes por razón de las cargas familiares.

ORDEN ASC/433/2007, de 23 de noviembre, por la que se establecen los criterios para determinar el importe de las prestaciones económicas del Sistema para la Autonomía y la Atención a la Dependencia (SAAD) en el ámbito territorial de Cataluña. (DOGC 5017, de 27/11/07)

La Ley 39/2006, de 14 de diciembre, de promoción de la autonomía personal y atención a las personas en situación de dependencia, creó tres prestaciones de carácter económico, concretamente la prestación económica vinculada al servicio, la prestación económica para cuidados en el entorno familiar y apoyo a los veladores no profesionales, y la prestación económica de asistencia personal.

La denominación, el contenido y los requisitos básicos de las prestaciones citadas están establecidos por los artículos 17, 18 y 19 de la Ley 39/2006, por lo que no es necesario un acto formal de creación para reconocer el derecho subjetivo correspondiente a las personas beneficiarias.

No obstante, las comunidades autónomas tienen potestad para establecer los requisitos específicos, las condiciones de acceso y, en particular, los criterios para determinar la cuantía de las prestaciones de acuerdo con los artículos 12 y 13 del Real decreto 727/2007, de 8 de junio. Además, la norma citada establece la obligación de aplicar coeficientes reductores sobre las cuantías vigentes en función de la capacidad económica del beneficiario.

Sin embargo, los importes estatales vigentes en la fecha de aprobación de la presente Orden, pese a que representan un avance sustancial sobre la situación precedente, aún no cubren el coste de los servicios objeto de financiación, particularmente la prestación vinculada al servicio. Por esta razón, la Orden también prevé la posibilidad de incrementar las prestaciones económicas del Sistema para la Autonomía y la Atención a la Dependencia (SAAD) en el marco de nivel adicional de protección previsto por el artículo 7.3 de la Ley 39/2006, que es competencia autonómica.

DECRETO 115/2007, de 22 de mayo, por el que se determinan los órganos de la Generalidad de Cataluña competentes para aplicar la Ley 39/2006, de 14 de diciembre, de promoción de la autonomía personal y atención a las personas en situación de dependencia. (DOGC 4890, de 24/05/07, corrección de errata en el DOGC núm. 4930)

De acuerdo con los artículos 27, 28 y 29 de la Ley 39/2006, de 14 de diciembre, de promoción de la autonomía personal y atención a las personas 
en situación de dependencia, la Generalitat de Cataluña es competente para determinar los órganos responsables de valorar y reconocer la situación de dependencia, establecer los servicios y las prestaciones correspondientes y, finalmente, aprobar el Programa Individual de Atención de las personas beneficiarias.

En la fecha de aprobación de este Decreto, el Consejo Territorial del Sistema para la Autonomía y la Atención de la Dependencia y el Gobierno del Estado aún no habían aprobado la normativa necesaria para estructurar y poner en pleno funcionamiento el sistema citado.

No obstante, la entrada en vigor del Real decreto 504/2007, de 20 de abril, por el que se aprueba el baremo de valoración de la situación de dependencia, permite iniciar los reconocimientos y declarar las situaciones de dependencia, imprescindibles para atender posteriormente las necesidades sociales urgentes que se deriven.

Antes de iniciar los reconocimientos, el Gobierno de la Generalitat de Cataluña debe determinar los órganos encargados de ejercer la competencia citada, tal como prevé el artículo 9.1 de la Ley 13/1989, de 14 de diciembre, de organización, procedimiento y régimen jurídico de la Administración de la Generalitat de Cataluña. Estos órganos podrán entonces iniciar la tramitación de las solicitudes, en el marco de la Ley 30/1992, de 26 de noviembre, de régimen jurídico de las administraciones públicas y el procedimiento administrativo común, de acuerdo con lo que establece el artículo 28.1 de la ley reguladora de las situaciones de dependencia.

ORDEN ASC/344/2008, de 14 de julio, por la que se regula la prestación económica de asistencia personal y se amplía el nivel de protección del Sistema Catalán de Autonomía y Atención a la Dependencia (SCAAD). (DOGC 5174, de 16/07/2008)

El artículo 19 de la Ley 39/2006, de 14 de diciembre, de promoción de la autonomía personal y atención a las personas en situación de dependencia, estableció una prestación económica de asistencia personal para las personas en situación de gran dependencia. El objetivo general de dicha prestación es facilitar la contratación de una asistencia personal, cuya finalidad será permitir el acceso a la educación y al trabajo, y una vida más autónoma en el ejercicio de las actividades básicas de la vida diaria.

Por otro lado, la disposición adicional primera de la Ley 12/2007, de 11 de octubre, de servicios sociales, entre las medidas para la promoción de la autonomía personal, contempla, en su apartado quinto, la regulación por reglamento de la figura del asistente personal. 
No obstante, las dos leyes citadas no concretan ni precisan los elementos esenciales de la prestación (objeto, modalidades, requisitos de las personas beneficiarias y del personal de asistencia, cuantías, compatibilidades e incompatibilidades), sin los cuales no es posible su aplicación ni tampoco el establecimiento de instrumentos jurídicos para garantizar la calidad del servicio y proteger los intereses de las personas beneficiarias.

Por esta razón, el Departament d'Acció Social i Ciutadania, de acuerdo con las necesidades existentes y teniendo en cuenta la evolución del sistema estatal de atención a la dependencia en los primeros meses de desarrollo, ha decidido regular normativamente, en una disposición específica, la prestación económica de asistencia personal.

La prestación, según la Ley estatal, procura facilitar la integración laboral y educativa de las personas en situación de gran dependencia y, en consecuencia, su autonomía y su integración social plena, sin que ello suponga una merma de su autonomía en las actividades básicas de la vida diaria.

Al mismo tiempo, la prestación se diseña en atención a las diferentes alternativas existentes de trabajo y educación, presencial y virtual, y a las necesidades de sus destinatarios. Por ello, la asistencia personal se estructura en dos modalidades. En primer lugar, la prestación de acompañamiento, de intensidad más limitada, para apoyar la integración laboral y educativa de carácter presencial. En segundo lugar, la prestación de apoyo personal, dotada de un nivel adicional de protección, para impulsar las modalidades laborales y educativas, virtuales y presenciales, en régimen de dedicación completa, y, también, la participación cívica de las personas beneficiarias.

Esta disposición normativa también regula los requisitos personales y materiales de la prestación, entre ellos la idoneidad y la formación del personal de asistencia, la cuantía de las prestaciones y el régimen de compatibilidades e incompatibilidades, razón por la que se modifican simultáneamente las órdenes ASC/433/2007, de 23 de noviembre (prestaciones económicas), y ASC/55/2008, de 12 de febrero (compatibilidades e incompatibilidades).

La Orden también establece un mecanismo para compensar la reducción de las prestaciones sociales análogas en los importes de las prestaciones económicas establecidas por el artículo 31 de la Ley estatal de dependencia. El mecanismo consiste en una garantía de importe mínimo, para evitar los casos en que la aplicación inevitable y obligada de la Ley deje las personas beneficiarias con menos recursos y sin ninguna cobertura adicional.

\section{CONCLUSIONES}

En primer lugar, la Ley 39/2006 tiene un impacto en el bienestar de las personas al garantizar el derecho a disfrutar de un conjunto de prestaciones 
que facilitan la autonomía en el desarrollo de las actividades básicas de la vida diaria. Antes de la Ley, las personas no tenían derecho a recibir una ayuda económica o un servicio público, sólo las personas con poca renta podían ser beneficiarias de los programas asistenciales que las comunidades autónomas habían desarrollado al respecto.

La Ley 39/2006 hará que el número de beneficiarios de las prestaciones públicas se multiplique por 8 , aproximadamente, dado que todos los ciudadanos, con independencia de su renta, podrán acceder a las prestaciones públicas. La evolución prevista del número de personas beneficiarias con dependencia protegibles por la Ley es pasar de 30.000 personas atendidas en 2007 a 250.000 en el año 2015.

El despliegue de la Ley implica un incremento importante de recursos económicos para atender a la población beneficiaria. Se prevé que el presupuesto necesario en el año 2015 se sitúe entre 1.800 y 2.000 millones de euros, aproximadamente, a precios constantes del año 2008. Esto implica que si tenemos en cuenta la previsión de la inflación a lo largo de estos años, los costes a precios reales se situarían entre 2.200 y 2.400 millones de euros.

El impacto económico de la Ley 39/2006 supondrá triplicar el gasto público actual pasando del $0,35 \%$ del PIB en el 2008 a casi el $1 \%$ del PIB en el 2015.

La Ley prevé prestaciones en servicios y prestaciones económicas, y la persona usuaria participa en la decisión final de la ayuda más adecuada a sus necesidades. El importe mediano de las prestaciones de la dependencia en el año 2015 será de 8.000 euros al año (precios constantes del año 2008).

Cataluña ha legislado prestaciones adicionales a las reguladas en la Ley 39/2006, lo cual supone que este incremento de gasto estará financiado sólo por la Generalitat (nivel adicional de financiación). En este grupo se encuentran: la prestación adicional a la prestación económica vinculada al servicio residencia, la prestación adicional a la prestación de la asistencia personal en su modalidad de apoyo personal e integración laboral y la prestación mínima garantizada.

También, Cataluña permite compatibilizar dos prestaciones con determinadas limitaciones de acuerdo con las necesidades de las personas usuarias, lo cual permite más variedad y flexibilidad en la oferta de las prestaciones de la dependencia. 
